\title{
Bounds for Departure from Normality and the Frobenius Norm of Matrix Eigenvalues
}

\author{
Steven L. Lee
}

\section{MANaGED BY}

MARTII MARIETIA ENERGY SYSTEMS, INC.

SOR THE UNITED STATES 
This report has been reproduced directly from the best available copy.

Available to DOE and DOE contractors from the Office of Scientific and Technical information, P.O. Box 62, Oak Ridge, TN 37831 ; prices available from (615) 576-8401, FTS 626-8401.

Available to the public from the National Technical Information Service, U.S. Department of Commerce, 5285 Port Royal Rd., Springfield, VA 22161.

This report was prepared as an account of work sponsored by an agency of the United States Government. Neither the United States Government nor any agency thereof, nor any of their employees, makes any warranty, express or implied, or assumes any legal liability or responsibility for the accuracy, completeness, or usefulness of any information, apparatus, product, or process disclosed, or represents that its use would not infringe privately owned rights. Reference herein to any specific commercial product, process, or service by trade name, trademark, manufacturer, or otherwise, does not necessarily constitute or imply its endorsement, recommendation, or favoring by the United States Government or any agency thereof. The views and opinions of authors expressed herein do not necessarily state or reflect those of the United States Government or any agency thereof. 


\section{DISCLAIMER}

\section{Portions of this document may be illegible in electronic image products. Images are produced from the best available original document.}




\title{
BOUNDS FOR DEPARTURE FROM NORMALITY AND THE FROBENIUS NORM OF MATRIX EIGENVALUES
}

\author{
Steven L. Lee
}

\author{
Mathematical Sciences Section \\ Oak Ridge National Laboratory \\ P.O. Box 2008, Bldg. 6012 \\ Oak Ridge, TN 37831-6367 \\ na.slee@na-net.ornl.gov
}

Date Published: December 1994

Research was supported by the Applied Mathematical Sciences Research Program of the Office of Energy Research, U.S. Department of Energy.

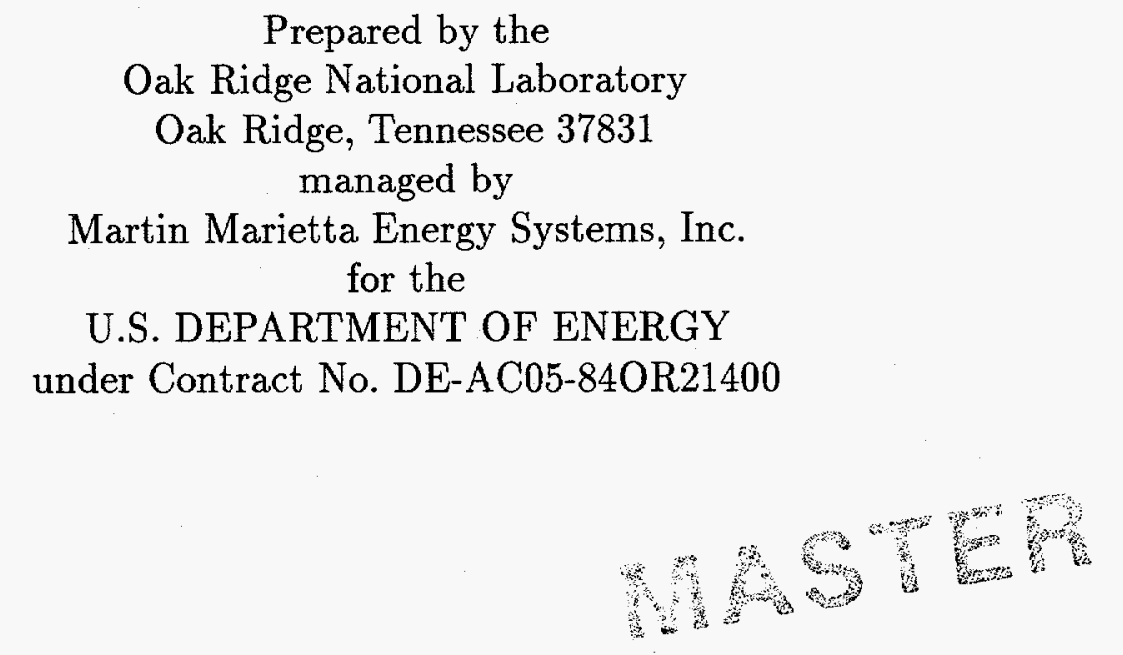




\section{Contents}

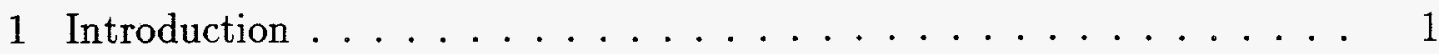

2 Preliminaries ..................... 2

3 Bounds for eigenvalues and departure from normality . . . . . . 4

3.1 Improved lower bounds . . . . . . . . . . . 5

3.2 Improved upper bounds $\ldots \ldots \ldots \ldots \ldots \ldots \ldots$

4 Main results . . . . . . . . . . . . . . . 7

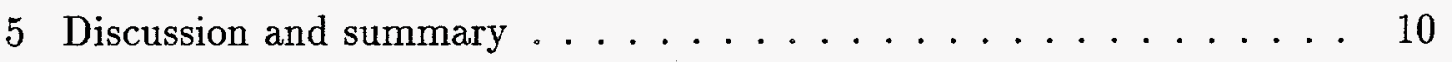

6 References ......................... . . 12 



\title{
BOUNDS FOR DEPARTURE FROM NORMALITY AND THE FROBENIUS NORM OF MATRIX EIGENVALUES
}

\author{
Steven L. Lee
}

\begin{abstract}
New lower and upper bounds for the departure from normality and the Frobenius norm of the eigenvalues of a matrix are given. The significant properties of these bounds are also described. For example, the upper bound for matrix eigenvalues improves upon the one derived by Kress, de Vries and Wegmann in [Lin. Alg. Appl., 8 (1974), pp. 109-120]. The upper bound for departure from normality is sharp for any matrix whose eigenvalues are collinear in the complex plane. Moreover, the latter formula. is a practical estimate that costs (at most) $2 m$ multiplications, where $m$ is the number of nonzeros in the matrix. In terms of applications, the results can be used to bound from above the sensitivity of eigenvalues to matrix perturbations or bound from below the condition number of the eigenbasis of a matrix.
\end{abstract}




\section{Introduction}

The departure from normality of a matrix, like the condition number of a matrix, is a real scalar that can be used to compute various matrix bounds. If $A$ is an $n \times n$ matrix, its departure from normality (in the Frobenius norm) is defined to be $[8]$

$$
\operatorname{dep}_{F}(A):=\left(\|A\|_{F}^{2}-\|\Lambda\|_{F}^{2}\right)^{1 / 2},
$$

where $\Lambda$ is a diagonal matrix whose entries are the eigenvalues, $\lambda_{k}$, of $A$. This measure of matrix nonnormality can be used to bound the spectral norm of matrix functions $[2,5]$, the sensitivity of eigenvalues to matrix perturbations $[8,16]$, and the distance to the closest normal matrix $[10,16]$, for example. It is impractical to compute $\operatorname{dep}_{F}(A)$ if $A$ is large and its eigenvalues are unknown. This difficulty motivates us to seek lower and upper bounds for $\operatorname{dep}_{F}(A)$ that are practical to compute or optimal in some sense.

In terms of eigenvalues, bounds for $\operatorname{dep}_{F}(A)$ can be used to obtain lower and upper bounds for

$$
\|\Lambda\|_{F}^{2}, \quad\|\operatorname{Re}(\Lambda)\|_{F}^{2}, \text { and }\|\operatorname{Im}(\Lambda)\|_{F}^{2},
$$

where $\operatorname{Re}(\Lambda)$ and $\operatorname{Im}(\Lambda)$ are the real and imaginary parts of $\Lambda$. In particular, such results can be obtained by substituting lower and upper bounds for $\operatorname{dep}_{F}(A)$ into [13]

$$
\begin{aligned}
\|\Lambda\|_{F}^{2} & =\|A\|_{F}^{2}-\operatorname{dep}_{F}^{2}(A), \\
\|\operatorname{Re}(\Lambda)\|_{F}^{2} & =\|M\|_{F}^{2}-\frac{1}{2} \operatorname{dep}_{F}^{2}(A), \\
\|\operatorname{Im}(\Lambda)\|_{F}^{2} & =\|N\|_{F}^{2}-\frac{1}{2} \operatorname{dep}_{F}^{2}(A)
\end{aligned}
$$

where

$$
M=\frac{1}{2}\left(A+A^{H}\right)
$$

and

$$
N=\frac{1}{2}\left(A-A^{H}\right)
$$

are the Hermitian and skew-Hermitian part of $A$, respectively. Upper bounds for $\|\Lambda\|_{F}^{2}$ can be used to bound the spectral radius [15] and the spread of a matrix [1]. Bounds for $\|\Lambda\|_{F}^{2}$ can also be used to compute or estimate lower bounds for the condition number of the eigenbasis of $A$ [11]. 
The outline of this paper is as follows. In $\S 2$, we give the notation, definitions, and observations that will be needed in later sections. In $\S 3$, we present various bounds for $\|\Lambda\|_{F}^{2}$ and $\operatorname{dep}_{F}^{2}(A)$, and show how they can be improved. In $\S 4$, we describe the significant properties of the newly improved bounds. In $\S 5$, we group the currently known a priori bounds for $\|\Lambda\|_{F}^{2}$ and $\operatorname{dep}_{F}^{2}(A)$ into two main categories, and then show that the new bounds are among the best available.

\section{Preliminaries}

Let $A=\left(a_{i j}\right)$ be an $n \times n$ matrix with conjugate transpose $A^{H}=\left(\bar{a}_{i j}\right)$ and Frobenius norm

$$
\|A\|_{F}^{2}:=\sum_{i, j}\left|a_{i j}\right|^{2}
$$

Also, recall that $A$ is normal if and only if (iff), for example, [7]

(9a) A has a complete, orthogonal set of eigenvectors,

(9b) $\|A\|_{F}=\|\Lambda\|_{F}=\sum\left|\lambda_{k}\right|^{2}$, or

(9c) $A^{H} A-A A^{H}=0$.

The set of normal matrices includes the Hermitian, skew-Hermitian, and unitary matrices and, in general, any matrix that is unitarily similar to a diagonal matrix. It is easily seen that $\operatorname{dep}_{F}(A)$ is invariant with respect to complex shifts and rotations. That is,

$$
\operatorname{dep}_{F}(A)=\operatorname{dep}_{F}\left(e^{-i \theta}(A-\alpha I)\right)
$$

for any complex scalar $\alpha$ and $0 \leq \theta<2 \pi$. For the Frobenius norm, we note that

$$
\left\|e^{-i \theta}(A-\alpha I)\right\|_{F}=\|A-\alpha I\|_{F}
$$

and

$$
\left\|(A-\alpha I)^{H_{(}}(A-\alpha I)-(A-\alpha I)(A-\alpha I)^{H}\right\|_{F}=\left\|A^{H} A-A A^{H}\right\|_{F}
$$

The simplification in (12) also holds when $A-\alpha I$ is replaced with $e^{-i \theta}(A-\alpha I)$. It is also easy to show that the quadratic function $\|A-\alpha I\|_{F}^{2}$ is minimized for $\alpha=\frac{\operatorname{tr}(A)}{n}$, where $\operatorname{tr}(A)$ is the trace of $A$. If $\operatorname{tr}(A)=0$, we shall say $A$ is a 
centered matrix. Centered matrices such as

$$
\tilde{A}=A-\frac{\operatorname{tr}(A)}{n} I
$$

and

$$
\tilde{\Lambda}=\Lambda-\frac{\operatorname{tr}(A)}{n} I
$$

will be denoted with a tilde accent. Finally, we give a lemma that relates the norm of the shifted matrices $A-\alpha I$ and $\Lambda-\alpha I$ to the norm of the centered matrices $\tilde{A}$ and $\tilde{\Lambda}$, respectively.

Lemma 2.1. For any $n \times n$ matrix $A$ and complex scalar $\alpha$,

$$
\|A-\alpha I\|_{F}^{2}=\|\tilde{A}\|_{F}^{2}+\frac{|\operatorname{tr}(A-\alpha I)|^{2}}{n}
$$

and

$$
\|\Lambda-\alpha I\|_{F}^{2}=\|\tilde{\Lambda}\|_{F}^{2}+\frac{|\operatorname{tr}(A-\alpha I)|^{2}}{n} .
$$

Proof: First, we relate the norm of $A$ to the norm of $\tilde{A}$. For $\sigma=\frac{\operatorname{tr}(A)}{n}$, we have

$$
\begin{aligned}
\|A\|_{F}^{2}-\|\tilde{A}\|_{F}^{2} & =\|A\|_{F}^{2}-\left\|A-\frac{\operatorname{tr}(A)}{n} I\right\|_{F}^{2} \\
& =\sum\left(\left|a_{i i}\right|^{2}\right)-\sum\left(\left|a_{i i}-\sigma\right|^{2}\right) \\
& =\sum\left(a_{i i}^{H} a_{i i}\right)-\sum\left[\left(a_{i i}-\sigma\right)^{H}\left(a_{i i}-\sigma\right)\right] \\
& =\sum\left(a_{i i}^{H} a_{i i}\right)-\sum\left[a_{i i}^{H} a_{i i}-a_{i i}^{H} \sigma-\sigma^{H} a_{i i}+\sigma^{H} \sigma\right] \\
& =\sigma \sum\left(a_{i i}^{H}\right)+\sigma^{H} \sum\left(a_{i i}\right)-n \sigma^{H} \sigma \\
& =\frac{\operatorname{tr}(A)}{n} \operatorname{tr}^{H}(A)+\frac{\operatorname{tr}^{H}(A)}{n} \operatorname{tr}(A)-n\left|\frac{\operatorname{tr}(A)}{n}\right|^{2} \\
& =\frac{2|\operatorname{tr}(A)|^{2}}{n}-\frac{|\operatorname{tr}(A)|^{2}}{n} \\
& =\frac{|\operatorname{tr}(A)|^{2}}{n} .
\end{aligned}
$$

If we now replace $A$ with $A-\alpha I$ on the right-hand side of (17) and (24), we obtain

$$
\|A-\alpha I\|_{F}^{2}-\left\|A-\alpha I-\frac{\operatorname{tr}(A-\alpha I)}{n} I\right\|_{F}^{2}=\frac{|\operatorname{tr}(A-\alpha I)|^{2}}{n},
$$


and the second term can be simplified via

$$
\begin{aligned}
\left\|A-\alpha I-\frac{\operatorname{tr}(A-\alpha I)}{n} I\right\|_{F} & =\left\|A-\alpha I-\frac{[\operatorname{tr}(A)-\operatorname{tr}(\alpha I)]}{n} I\right\|_{F}^{2} \\
& =\left\|A-\alpha I-\frac{\operatorname{tr}(A)}{n} I+\frac{n \alpha}{n} I\right\|_{F}^{2} \\
& =\left\|A-\frac{\operatorname{tr}(A)}{n} I\right\|_{F}^{2} \\
& =\|\tilde{A}\|_{F}^{2}
\end{aligned}
$$

to obtain (15). The second equality (16) can also be proved in this manner.

\section{Bounds for eigenvalues and departure from normality}

We now present several bounds for $\|\Lambda\|_{F}^{2}$ and $\operatorname{dep}_{F}^{2}(A)$, along with their important properties. An upper bound for $\|\Lambda\|_{F}^{2}$ is given by Kress, de Vries, and Wegmann in [9]. Moreover, the authors exhibit nonnormal matrices for which the bound is sharp, and prove that the upper bound is the best possible in terms of $\|A\|_{F}$ and $\left\|A^{H} A-A A^{H}\right\|_{F}$.

Theorem 3.1. [9, Thm. 1] For nonnormal $A$ there holds

$$
\|\Lambda\|_{F}^{2} \leq\left(\|A\|_{F}^{4}-\frac{1}{2}\left\|A^{H} A-A A^{H}\right\|_{F}^{2}\right)^{1 / 2}
$$

with equality iff

$$
A=\gamma\left(v w^{H}+r w v^{H}\right)
$$

where $\gamma$ is a nonzero complex scalar, $0 \leq r<1$ is a real scalar, and where $v, w$ are orthonormal vectors.

A practical lower bound for $\|\Lambda\|_{F}^{2}[5]$,

$$
\left|\operatorname{tr}\left(A^{2}\right)\right| \leq\|\Lambda\|_{F}^{2}
$$

comes from the triangle inequality applied to the eigenvalues of $A^{2}$ :

$$
\begin{aligned}
\left|\operatorname{tr}\left(A^{2}\right)\right| & =\left|\operatorname{tr}\left(\Lambda^{2}\right)\right|=\left|\sum \lambda_{i}^{2}\right|=\left|\lambda_{1}^{2}+\cdots+\lambda_{n}^{2}\right| \\
& \leq\left|\lambda_{1}^{2}\right|+\cdots+\left|\lambda_{n}^{2}\right|=\left|\lambda_{1}\right|^{2}+\cdots+\left|\lambda_{n}\right|^{2}=\|\Lambda\|_{F}^{2} .
\end{aligned}
$$


The lower bound is sharp iff 0 and the eigenvalues of $A$ are collinear. Moreover, the bound is cheap to compute since only the diagonal of $A^{2}$ is needed. This diagonal can be computed with (at most) $m$ multiplications, where $m$ is the number of nonzeros in $A$.

The lower bound [17] and the upper bound [5] for $\operatorname{dep}_{F}^{2}(A)$,

$$
\|A\|_{F}^{2}-\left(\|A\|_{F}^{4}-\frac{1}{2}\left\|A^{H} A-A A^{H}\right\|_{F}^{2}\right)^{1 / 2} \leq \operatorname{dep}_{F}^{2}(A) \leq\|A\|_{F}^{2}-\left|\operatorname{tr}\left(A^{2}\right)\right|
$$

can be obtained by substituting (30) and (32) into (1). The upper bound in (35) is sharp iff 0 and the eigenvalues of $A$ are collinear, and it can be computed with (at most) $2 m$ multiplications. The lower bound is an $O\left(n^{3}\right)$ computation that is sharp if $A$ is normal, or satisfies condition (31). This lower bound inherits the properties of the upper bound (30) via (1); thus, it is the best possible in terms of $\|A\|_{F}$ and $\left\|A^{H} A-A A^{H}\right\|_{F}$.

In $\S 3.1$, we will strengthen the lower bounds for $\operatorname{dep}_{F}^{2}(A)$ and $\|\Lambda\|_{F}^{2}$ in (35) and (34). In $\S 3.2$, these improved lower bounds will be substituted into (3) and (1), respectively, to obtain tighter upper bounds for $\|\Lambda\|_{F}^{2}$ and $\operatorname{dep}_{F}^{2}(A)$.

\subsection{Improved lower bounds}

The value of $\operatorname{dep}_{F}(A)$ is invariant with respect to the shift parameter $\alpha$; see (10). We now show how this free parameter can be used to maximize the lower bound in (35). For normal matrices, the lower bound

$$
\|A-\alpha I\|_{F}^{2}-\left(\|A-\alpha I\|_{F}^{4}-\frac{1}{2}\left\|A^{H} A-A A^{H}\right\|_{F}^{2}\right)^{1 / 2} \leq \operatorname{dep}_{F}^{2}(A-\alpha I)=\operatorname{dep}_{F}^{2}(A)
$$

is zero for any choice of $\alpha$. For nonnormal matrices, however, there is a unique value of $\alpha$ that maximizes (36). In particular, by substituting (15) into (36), we seek to maximize the function

$$
f(z(\alpha))=\left(\beta^{2}+z^{2}(\alpha)\right)-\left[\left(\beta^{2}+z^{2}(\alpha)\right)^{2}-\frac{1}{2} K^{2}\right]^{1 / 2}
$$

where

$$
z^{2}(\alpha)=\frac{|\operatorname{tr}(A-\alpha I)|^{2}}{n}
$$


and

$$
\beta^{2}=\|\tilde{A}\|_{F}^{2}, \quad K^{2}=\left\|A^{H} A-A A^{H}\right\|_{F}^{2}>0 .
$$

By solving

$$
\frac{d f}{d z}=2 z\left(1-\left(\beta^{2}+z^{2}\right)\left[\left(\beta^{2}+z^{2}\right)^{2}-\frac{1}{2} K^{2}\right]^{-1 / 2}\right)=0
$$

we find that the unique solution $z=0$ is a global maximum since

$$
\frac{d^{2} f}{d z^{2}}(0)=2\left(1-\frac{\beta^{2}}{\left(\beta^{4}-\frac{1}{2} K^{2}\right)^{1 / 2}}\right)<0 .
$$

By solving

$$
z(\alpha)=\frac{|\operatorname{tr}(A-\alpha I)|}{n^{1 / 2}}=0
$$

we find that the lower bound is maximized for $\alpha=\frac{\operatorname{tr}(A)}{n}$.

Lemma 3.2. For any $n \times n \operatorname{matrix} A$,

$$
\operatorname{dep}_{F}^{2}(A) \geq\|A-\alpha I\|_{F}^{2}-\left(\|A-\alpha I\|_{F}^{4}-\frac{1}{2}\left\|A^{H} A-A A^{H}\right\|_{F}^{2}\right)^{1 / 2}
$$

where the lower bound is maximized for $\alpha=\frac{\operatorname{tr}(A)}{n}$.

Proof: The lemma follows via (37)-(42).

An improved lower bound for $\|\Lambda\|_{F}^{2}$ is less troublesome to obtain.

Lemma 3.3. For any $n \times n$ matrix $A$,

$$
\|\Lambda\|_{F}^{2} \geq\left|\operatorname{tr}\left(\tilde{A}^{2}\right)\right|+\frac{|\operatorname{tr}(A)|^{2}}{n}
$$

where $\tilde{A}=A-\frac{\operatorname{tr}(A)}{n} I$.

Proof: As in (33)-(34), we begin by applying the triangle inequality to the eigenvalues of $\tilde{A}^{2}$,

$$
\left|\operatorname{tr}\left(\tilde{A}^{2}\right)\right|=\left|\operatorname{tr}\left(\tilde{\Lambda}^{2}\right)\right| \leq\left\|\tilde{\Lambda}^{2}\right\|_{F}=\|\tilde{\Lambda}\|_{F}^{2} .
$$


The lemma is obtained by substituting this lower bound for $\|\tilde{\Lambda}\|_{F}^{2}$ into

$$
\|\Lambda\|_{F}^{2}=\|\tilde{\Lambda}\|_{F}^{2}+\frac{|\operatorname{tr}(A)|^{2}}{n}
$$

Note that (46) is the same as (16) with $\alpha=0$.

\subsection{Improved upper bounds}

For the $\|\Lambda\|_{F}^{2}$ upper bound, we have

$$
\begin{aligned}
\|\Lambda\|_{F}^{2} & =\|A\|_{F}^{2}-\operatorname{dep}_{F}^{2}(A) \\
& \leq\|A\|_{F}^{2}-\left(\|\tilde{A}\|_{F}^{2}-\left(\|\tilde{A}\|_{F}^{4}-\frac{1}{2}\left\|A^{H} A-A A^{H}\right\|_{F}^{2}\right)^{1 / 2}\right) .
\end{aligned}
$$

Equation (15), with $\alpha=0$, shows that $\|A\|_{F}^{2}-\|\tilde{A}\|_{F}^{2}$ simplifies to $\frac{|\operatorname{tr}(A)|^{2}}{n}$.

Lemma 3.4. For any $n \times n$ matrix $A$,

$$
\|\Lambda\|_{F}^{2} \leq\left(\|\tilde{A}\|_{F}^{4}-\frac{1}{2}\left\|A^{H} A-A A^{H}\right\|_{F}^{2}\right)^{1 / 2}+\frac{|\operatorname{tr}(A)|^{2}}{n}
$$

where $\tilde{A}=A-\frac{\operatorname{tr}(A)}{n} I$.

For the $\operatorname{dep}_{F}^{2}(A)$ upper bound, we can substitute $\|\tilde{\Lambda}\|_{F}^{2} \geq\left|\operatorname{tr}\left(\tilde{A}^{2}\right)\right|$ into

$$
\operatorname{dep}_{F}^{2}(A)=\operatorname{dep}_{F}^{2}(\tilde{A})=\|\tilde{A}\|_{F}^{2}-\|\tilde{\Lambda}\|_{F}^{2}
$$

to obtain the following lemma.

Lemma 3.5. For any $n \times n$ matrix $A$,

$$
\operatorname{dep}_{F}^{2}(A) \leq\|\tilde{A}\|_{F}^{2}-\left|\operatorname{tr}\left(\tilde{A}^{2}\right)\right|
$$

where $\tilde{A}=A-\frac{\operatorname{tr}(A)}{n} I$.

\section{Main results}

In this section, we establish the significant properties of the four bounds given in $\S 3.1$ and $\S 3.2$. To begin, recall that the lower bound for $\operatorname{dep}_{F}^{2}(A)$ and, in 
turn, the upper bound for $\|\Lambda\|_{F}^{2}$ were optimized via the complex shift $\alpha=\frac{\operatorname{tr}(A)}{n}$. Moreover, the latter derivation (47)-(48) shows that if the $\operatorname{dep}_{F}^{2}(A)$ lower bound is sharp then so is the $\|\Lambda\|_{F}^{2}$ upper bound. The $\operatorname{dep}_{F}^{2}(A)$ lower bound in (35) is sharp for any nonnormal matrix that satisfies condition (31). The improved $\operatorname{dep}_{F}^{2}(A)$ lower bound (43) is unaffected by complex shifts; thus, it is sharp for

$$
A=\gamma\left(v w^{H}+r w v^{H}\right)-\sigma I
$$

for any choice of the scalar $\sigma$. Note that we have

$$
\alpha=\frac{\operatorname{tr}\left(\gamma\left(v w^{H}+r w v^{H}\right)-\sigma I\right)}{n}=-\sigma
$$

and that the shift $\alpha$ in (43) cancels the arbitrary shift $\sigma$. The improved bound is also unaffected by rotations. We summarize the above results as follows.

Theorem 4.1. For any $n \times n$ matrix $A$,

$$
\operatorname{dep}_{F}^{2}(A) \geq\|\tilde{A}\|_{F}^{2}-\left(\|\tilde{A}\|_{F}^{4}-\frac{1}{2}\left\|A^{H} A-A A^{H}\right\|_{F}^{2}\right)^{1 / 2}
$$

and

$$
\|\Lambda\|_{F}^{2} \leq\left(\|\tilde{A}\|_{F}^{4}-\frac{1}{2}\left\|A^{H} A-A A^{H}\right\|_{F}^{2}\right)^{1 / 2}+\frac{|\operatorname{tr}(A)|^{2}}{n},
$$

where $\tilde{A}=A-\frac{\operatorname{tr}(A)}{n} I$. The bounds are sharp iff

$$
A=e^{-i \theta}\left(\gamma\left(v w^{H}+r w v^{H}\right)-\sigma I\right)
$$

where $\gamma, r$ and $\sigma$ are complex scalars, $0 \leq \theta<2 \pi$, and where $v$, $w$ are orthonormal vectors.

We will now prove that the other two bounds (44) and (51) are sharp iff the eigenvalues of $A$ are collinear in the complex plane. Before doing so, we must establish a natural measure of the noncollinearity of matrix eigenvalues. One approach is to define "departure from collinearity" as

$$
\operatorname{depcol}(A):=\sum\left|d_{k}\right|^{2}
$$

where $\left|d_{k}\right|$ is the perpendicular distance from $\lambda_{k}$ to the total least squares (TLS) fit of the eigenvalues of $A$. Recall that a TLS fit minimizes the sum of the squares 
of the perpendicular distances from the points to the fitted line, and that $\sum\left|d_{k}\right|^{2}$ is the TLS error [6]. Given the definition (57), we find $\operatorname{depcol}(A)$ to be a sensible metric for quantifying departure from collinearity, especially since $\operatorname{dep} \operatorname{col}(A)=0$ iff $A$ has collinear eigenvalues.

A useful result concerning TLS error and departure from collinearity follows from [12, Thm. 2.2].

Theorem 4.2. Given the complex numbers $z_{k}, k=1, \cdots, n$, let $\bar{z}=\frac{1}{n} \sum z_{k}$ so that

$$
\tilde{z}_{k}=z_{k}-\bar{z}
$$

The error for the total least squares fit is

$$
\sum\left|d_{k}\right|^{2}=\frac{1}{2}\left(\sum\left|\tilde{z}_{k}\right|^{2}-\left|\sum \tilde{z}_{k}^{2}\right|\right)
$$

where $\left|d_{k}\right|$ is the perpendicular distance from $z_{k}$ to the fit.

In the context of matrix eigenvalues, (59) yields

$$
\operatorname{depcol}(A)=\frac{1}{2}\left(\|\tilde{\Lambda}\|_{F}^{2}-\left|\operatorname{tr}\left(\tilde{A}^{2}\right)\right|\right)
$$

If we arrange $(60)$ as

$$
\|\tilde{\Lambda}\|_{F}^{2}=\left|\operatorname{tr}\left(\tilde{A}^{2}\right)\right|+2 \operatorname{depcol}(A)
$$

and substitute into (46) and (50), we obtain

$$
\|\Lambda\|_{F}^{2}=\left|\operatorname{tr}\left(\tilde{A}^{2}\right)\right|+2 \operatorname{depcol}(A)+\frac{|\operatorname{tr}(A)|^{2}}{n}
$$

and

$$
\operatorname{dep}_{F}^{2}(A)=\|\tilde{A}\|_{F}^{2}-\left(\left|\operatorname{tr}\left(\tilde{A}^{2}\right)\right|+2 \operatorname{depcol}(A)\right) .
$$

Note that the bounds in Lemmas 3.3 and 3.5 are special cases of (62) and (63).

Theorem 4.3. For any $n \times n$ matrix $A$,

$$
\|\Lambda\|_{F}^{2} \geq\left|\operatorname{tr}\left(\tilde{A}^{2}\right)\right|+\frac{|\operatorname{tr}(A)|^{2}}{n}
$$


and

$$
\operatorname{dep}_{F}^{2}(A) \leq\|\tilde{A}\|_{F}^{2}-\left|\operatorname{tr}\left(\tilde{A}^{2}\right)\right|,
$$

where $\tilde{A}=A-\frac{\operatorname{tr}(A)}{n} I$. The bounds are sharp iff the eigenvalues of $A$ are collinear in the complex plane. Moreover,

$$
\|\Lambda\|_{F}^{2} \approx\left|\operatorname{tr}\left(\tilde{A}^{2}\right)\right|+\frac{|\operatorname{tr}(A)|^{2}}{n}
$$

and

$$
\operatorname{dep}_{F}^{2}(A) \approx\|\tilde{A}\|_{F}^{2}-\left|\operatorname{tr}\left(\tilde{A}^{2}\right)\right|
$$

iff the eigenvalues of $A$ are relatively close to being collinear.

Proof: The bounds (64)-(65) are obtained from (62)-(63) by dropping the term $2 \operatorname{depcol}(A)$. These bounds are sharp iff $\operatorname{dep} \operatorname{col}(A)=0$; that is, iff the eigenvalues of $A$ are collinear. Finally, the bounds are good estimates when the neglected term $\operatorname{dep} \operatorname{col}(A)$ is relatively small.

\section{Discussion and summary}

To the best of our knowledge, a priori bounds for $\|\Lambda\|_{F}^{2}$ and $\operatorname{dep}_{F}^{2}(A)$ fall into one of two distinct categories. The bounds in the first category are based on computing the Frobenius norm of the commutator $A^{H} A-A A^{H}[3,4,8,14,17]$. The bounds in the second category are based on inequalities that are sharp iff the eigenvalues of $A$ have a certain alignment in the complex plane [5,13]. For each of these categories, we now give the best available bounds known to us at this time.

Bounds based on $\left\|A^{H} A-A A^{H}\right\|_{F}$

$$
\begin{aligned}
\|\Lambda\|_{F}^{2} & \geq\|A\|_{F}^{2}-\left(\frac{n^{3}-n}{12}\right)^{1 / 2}\left(\left\|A^{H} A-A A^{H}\right\|_{F}\right) \\
\|\Lambda\|_{F}^{2} & \leq\left(\|\tilde{A}\|_{F}^{4}-\frac{1}{2}\left\|A^{H} A-A A^{H}\right\|_{F}^{2}\right)^{1 / 2}+\frac{|\operatorname{tr}(A)|^{2}}{n} \\
\operatorname{dep}_{F}^{2}(A) & \geq\|\tilde{A}\|_{F}^{2}-\left(\|\tilde{A}\|_{F}^{4}-\frac{1}{2}\left\|A^{H} A-A A^{H}\right\|_{F}^{2}\right)^{1 / 2}
\end{aligned}
$$




$$
\operatorname{dep}_{F}^{2}(A) \leq\left(\frac{n^{3}-n}{12}\right)^{1 / 2}\left(\left\|A^{H} A-A A^{H}\right\|_{F}\right)
$$

REMARKS. The lower bound (68) is the counterpart to the upper bound (71) of Henrici [8, Thm. 1]. The bounds (69)-(70) are given in Theorem 4.1. Note that Sun's lower bound (35) is the best possible in terms of $\left\|A^{H} A-A A^{H}\right\|_{F}$ and $\|A\|_{F}$; thus it is stronger than the bounds in $[3,4]$. The bound (70) improves upon Sun's lower bound, and it is also stronger than the one in [14].

\section{Bounds based on eigenvalue alignment}

$$
\begin{aligned}
\|\Lambda\|_{F}^{2} & =\left|\operatorname{tr}\left(\tilde{A}^{2}\right)\right|+2 \operatorname{dep} \operatorname{col}(A)+\frac{|\operatorname{tr}(A)|^{2}}{n} \geq\left|\operatorname{tr}\left(\tilde{A}^{2}\right)\right|+\frac{|\operatorname{tr}(A)|^{2}}{n} \\
\operatorname{dep}_{F}^{2}(A) & =\|\tilde{A}\|_{F}^{2}-\left(\left|\operatorname{tr}\left(\tilde{A}^{2}\right)\right|+2 \operatorname{dep} \operatorname{col}(A)\right) \leq\|\tilde{A}\|_{F}^{2}-\left|\operatorname{tr}\left(\tilde{A}^{2}\right)\right|
\end{aligned}
$$

REMARKs. The new bounds (72)-(73) are sharp iff the eigenvalues of $A$ are collinear. In contrast, note that for $\alpha=\frac{\operatorname{tr}(A)}{n}$, we have [13, Thm. 3.2]

$$
\operatorname{dep}_{F}^{2}(A) \leq 2 \min \left\{\|M-\operatorname{Re}(\alpha) I\|_{F}^{2},\|N-i \operatorname{Im}(\alpha) I\|_{F}^{2}\right\}
$$

and its (unsimplified) counterpart

$$
\|\Lambda\|_{F}^{2} \geq\|A\|_{F}^{2}-2 \min \left\{\|M-\operatorname{Re}(\alpha) I\|_{F}^{2},\|N-i \operatorname{Im}(\alpha) I\|_{F}^{2}\right\} .
$$

Unfortunately, the bounds (74)-(75) are sharp only when the eigenvalues are horizontally or vertically aligned in the complex plane. Furthermore, the bounds in (72)-(73) are half as expensive to compute as those in (74)-(75). Despite these shortcomings, the latter bounds are useful and have some noteworthy properties. In particular, the bounds in (72)-(73) and those in (74)-(75) yield the same values if $A$ is a real matrix. We also remark that (74) explicitly bounds matrix nonnormality in terms of the nonsymmetry of $A$.

Besides their practicality, the estimates (72)-(73) are also appealing because they sometimes enable us to precisely compute $\|\Lambda\|_{F}^{2}$ and $\operatorname{dep}_{F}^{2}(A)$ for matrices with extremely sensitive eigenvalues. For example, consider the $n \times n$ matrix

$$
\widehat{W}_{n}=U^{H} W_{n} U
$$


where $\widehat{W}_{n}$ is dense and unitarily similar to the Wilkinson matrix [18, pg. 90]

$$
W_{n}=\left[\begin{array}{ccccc}
n & n & & & \\
& n-1 & n & & \\
& & \ddots & \ddots & \\
& & & 2 & n \\
& & & & 1
\end{array}\right]
$$

where $n=20$. The eigenvalues of $\widehat{W}_{n}$ are real, and the interior eigenvalues are notoriously difficult to compute for $n \gg 20$. Thus, we cannot directly compute $\|\Lambda\|_{F}^{2}$ for, say, $\widehat{W}_{50}$ due to these eigenvalue sensitivities. However, we can accurately obtain $\|\Lambda\|_{F}^{2}$ and $\operatorname{dep}_{F}^{2}(A)$ for $\widehat{W}_{50}$ via (72)-(73) since the sharpness of these formulas (modulo rounding errors) only depends upon eigenvalue collinearity - not eigenvalue sensitivity.

To summarize, we have developed several new and improved bounds for $\operatorname{dep}_{F}^{2}(A)$ and $\|\Lambda\|_{F}^{2}$, and described their significant properties. We have also grouped these and the other known a priori bounds for $\operatorname{dep}_{F}^{2}(A)$ and $\|\Lambda\|_{F}^{2}$ into two categories. Within each category, we have given the best available bounds. The bounds based on $\left\|A^{H} A-A A^{H}\right\|_{F}$ have an important property: they reduce to zero if $A$ is normal. Unfortunately, such bounds are often weak, and impractical to compute if $A$ is large. On the other hand, the bounds based on eigenvalue alignment are often good estimates (e.g., [13, Table 1]), and they are practical to compute if $A$ is large and sparse. A minor drawback is that these bounds only reduce to zero for normal matrices with collinear eigenvalues (e.g., Hermitian and skew-Hermitian matrices). Theorem 4.1, Theorem 4.3 and [8, Thm. 1] describe the nonnormal matrices for which the bounds in (68)-(73) are sharp. The significance of our results are described in $\S 1$.

\section{References}

[1] N. A. Derzko and A. M. Pfeffer. Bounds for the spectral radius of a matrix. Math. Comp., 19:62-67, 1965.

[2] J. Descloux. Bounds for the spectral norm of functions of matrices. Numer. Math., pages 185-190, 1963. 
[3] P. J. Eberlein. On measures of non-normality for matrices. Amer. Math. Mon., 72:995-996, 1965.

[4] L. Elsner and M. H. C. Paardekooper. On measures of nonnormality of matrices. Linear Algebra Appl., 92:107-124, 1987.

[5] M. Gil'. Estimate for the norm of matrix-valued functions. Linear Multilinear Algebra, 35:65-73, 1993.

[6] G. H. Golub and C. F. Van Loan. An analysis of the total least squares problem. SIAM J. Numer. Anal., 17:883-893, 1980.

[7] R. Grone, C. R. Johnson, E. M. Sá, and H. Wolkowicz. Normal matrices. Linear Algebra Appl., 87:213-225, 1987.

[8] P. Henrici. Bounds for iterates, inverses, spectral variation and fields of values of non-normal matrices. Numer. Math., 4:24-40, 1962.

[9] R. Kress, H. L. de Vries, and R. Wegmann. On nonnormal matrices. Linear Algebra Appl., 8:109-120, 1974.

[10] L. László. An attainable lower bound for the best normal approximation. SIAM J. Matrix Anal. Appl., 15:1035-1043, 1994.

[11] S. L. Lee. On the eigenvalue sensitivity of nonnormal matrices. Technical report, Oak Ridge National Laboratory. In preparation.

[12] S. L. Lee. A note on the total least squares problems for coplanar points. Technical report TM-12852, Oak Ridge National Laboratory, September 1994.

[13] S. L. Lee. A practical upper bound for departure from normality. SIAM J. Matrix Anal. Appl., 16(2), April 1995. To appear.

[14] G. Loizou. Nonnormality and Jordan condition numbers of matrices. $J$. Assoc. Comput. Mach., 16(4):580-584, October 1969.

[15] L. Mirsky. The spread of a matrix. Mathematika, 3:127-130, 1956.

[16] A. Ruhe. Closest normal matrix finally found! BIT, 27:585-598, 1987. 
[17] J.-G. Sun. Matrix Perturbation Analysis. Chinese Academic Press, 1987. In Chinese.

[18] J. H. Wilkinson. The Algebraic Eigenvalue Problem. Claredon Press, Oxford, England, 1965. 
ORNL/TM-12853

\section{INTERNAL DISTRIBUTION}

1. B.R. Appleton

2. T.S. Darland

3. E.F. D'Azevedo

4. J.M. Donato

5. J.J. Dongarra

6. J.B. Drake

7. G.A. Geist

8-12. M.R. Leuze

13. N.M. Nachtigal

14-18. E.G. Ng

19. C.E. Oliver

20. P.M. Papadopoulos

\author{
21-25. S.A. Raby \\ 26. C.H. Romine \\ 27. T.H. Rowan \\ 28. B.D. Semeraro \\ 29-33. R.F. Sincovec \\ 34-38. R.C. Ward \\ 39. Central Research Library \\ 40. ORNL Patent Office \\ 41. K-25 Appl Tech Library \\ 42. Y-12 Technical Library \\ 43. Lab Records Dept - RC \\ 44-45. Laboratory Records Dept
}

\section{EXTERNAL DISTRIBUTION}

46. Loyce Adams, Dept. of Applied Mathematics, FS-20, University of Washington, Seattle, WA 98195

47. Fernando Alvarado, University of Wisconsin, 1425 Johnson Drive, Madison, WI 53706-1607

48. Greg Ammar, Dept. of Mathematical Sciences, Northern Illinois University, DeKalb, IL 60115

49. Mario Arioli, IAN-CNR, c/o Dipartimento di Matematica, 209, via Abbiategrasso, 27100 Pavia, Italy

50. Steven Ashby, Lawrence Livermore National Lab, P.O. Box 808, L-316, Livermore, CA 94551-0808

51. Donald M. Austin, 1100 Washington Ave. South, Room 101, University of Minnesota, Minneapolis, MN 55455

52. Owe Axelsson, Faculty of Mathematics and Informatics, University of Nijmegen, NL-6525 ED Nijmengen The Netherlands

53. Zhaojun Bai, Department of Mathematics, University of Kentucky, Lexington, KY 40506

54. Jesse Barlow, Dept. of Computer Science and Engineering, Pennsylvania State University, 220 Pond Laboratory, University Park, PA 16802-6106

55. Edward H. Barsis, Sandia National Laboratories, Computer Science and Mathematics, P.O. Box 5800, Albuquerque, NM 87185

56. Michael Berry, Department of Computer Science, 114 Ayres Hall, University of Tennessee, Knoxville, TN 37996-1301 
57. Rob Bisseling, University of Utrecht, Department of Mathematics, P.O. Box 80010, NL-3508 TA Utrecht, The Netherlands

58. Ake Bjorck, Dept. of Mathematics, Linkoping University, S-58183 Linkoping, Sweden

59. Petter Bjorstad, Institutt for Informatikk, Hoyteknologisenteret, N-5020 Bergen, Norway

60. Thierry Braconnier, 42 Ave G. Coriolis, 31057 Toulouse Cedex, France

61. Randall Bramley, Department of Computer Science, 215 Lindley Hall, Indiana University, IN 47405

62. Roger W. Brockett, Harvard University, 29 Oxford Street, Cambridge, MA 021382901

63. Peter Brown, Lawrence Livermore National Laboratory, P.O. Box 808, L-316, Livermore, CA 94551

64. Richard Brualdi, Dept. of Mathematics, 480 Lincoln Drive, Madison, WI 53706

65. Ralph Byers, 405 Snow Hall, Department of Mathematics, University of Kansas, Lawrence, KS 66045

66. Daniela Calvetti, Department of Mathematics, Stevens Institute of Technology, Hoboken, NJ 07030

67. Tony Chan, Dept. of Mathematics, University of California, Los Angeles, 405 Hilgard Avenue, Los Angeles, CA 90024-1555

68. Françoise Chaitin-Chatelin, 18 Rue Pierre Arnoux, 92190 Meudon, France

69. Paul Concus, Mathematics and Computing, Lawrence Berkeley Laboratory, 50A/2129, University of California, Berkeley, CA 94720

70. Jane Cullum, IBM T.J. Watson Research Center, P.O. Box 218, Yorktown Heights, NY 10598

71. Biswa Datta, Dept. of Mathematical Sciences, Northern Illinois University, DeKalb, IL 60115

72. Tim Davis, E301 CSE, University of Florida, Gainesville, FL 32611-2024

73. James Demmel, Dept. of Computer Science, University of California, 571 Evans Hall, Berkeley, CA 94720

74. John Dorning, Dept. of Nuclear Engineering Physics, Nuclear Reactor Facility, University of Virginia, Charlottesville, VA 22903-2442

75. Milo Dorr, Lawrence Livermore National Laboratory, P.O. Box 808, L-316, Livermore, CA 94551

76. Craig Douglas, 8 South Street, Cos Cob, CT 06807-1618

77. Tony Drummond, 42 Ave G. Coriolis, 31057 Toulouse Cedex, France

78. Donald J. Dudziak, Dept. of Nuclear Engineering, 110B Burlington Engineering Labs, North Carolina State University, Raleigh, NC 27695-7909

79. Iain Duff, Rutherford Appleton Laboratory, Didcot, Oxon 0X11OQX, England 
80. Patricia Eberlein, Department of Computer Science, State University of New York, 226 Bell Hall, Buffalo, NY 14260

81. Michael Eiermann, Institut für Angewandte Mathematik II, TU Bergakademie Freiberg, D-09596 Freiberg, Federal Republic of Germany

82. Stanley C. Eisenstat, Department of Computer Science, Yale University, P.O. Box 2158, New Haven, CT 06520

83. Tommy Elfving, University of Linkoping, MAI, 58183 Linkoping, Sweden

84. Howard C. Elman, Dept. of Computer Science, University of Maryland, College Park, MD 20742

85. Richard E. Ewing, Department of Mathematics, Texas A\&M University, College Station, TX 77843

86. Robert Falgout, Lawrence Livermore National Laboratory, P.O. Box 808, L-419, Livermore, CA 94551

87. Bernd Fischer, Universität Hamburg, Institut fur Angewandte Mathematik, Bundersstrasse 55, D-2000 Hamburg 13, Federal Republic of Germany

88. Geoffrey C. Fox, Northeast Parallel Architectures Center, 111 College Place, Syracuse University, Syracuse, NY 13244-4100

89. Valérie Frayssé, CERFACS, 42 Ave G. Coriolis, 31057 Toulouse Cedex, France

90. Roland Freund, AT\&T Bell Labs, Room 2C-420, 600 Mountain Avenue, Murray Hill, NJ 07974-0636

91. Robert Funderlic, Computer Science Department, Box 8206, 228 Withers Hall, North Carolina State University, Raleigh, NC 27695-8206

92. Walter Gander, Institut für Wissenschaftliches Rechnen, ETH-Zentrum, CH-8092 Zurich, Switzerland

93. C. William Gear, NEC Research Institute, 4 Independence Way, Princeton, NJ 08540-6621

94. J. Alan George, Vice President, Academic and Provost, Needles Hall, University of Waterloo, Waterloo, Ontario, Canada N2L 3G1

95. John Gilbert, Xerox Palo Alto Research Center, 3333 Coyote Hill Road, Palo Alto, CA 94304

96. Luc Giraud, 42 Ave G. Coriolis, 31057 Toulouse Cedex, France

97. Gene H. Golub, Dept. of Computer Science, Stanford University, Stanford, CA 94305

98. Nick Gould, Central Computing Department, Rutherford Appleton Laboratory, Chilton, Oxfordshire, 0X11OQX, England

99. Serge Gratton, 42 Ave G. Coriolis, 31057 Toulouse Cedex, France

100. Joe Grcar, Sandia National Laboratories, Livermore, CA 94551-0969

101. Anne Greenbaum, Courant Institute, New York University, 251 Mercer Street, New York, NY 10012-1185

102. John Gustafson, Ames Laboratory, Iowa State University, Ames, IA 50011 
103. Martin Gutknecht, IPS Supercomputing, ETH-Zentrum, CH-8092 Zurich, Switzerland

104. Christian Halloy, Assistant Director of JICS, 104 South College, Joint Institute for Computational Science, University of Tennessee, Knoxville, TN 37996-1301

105. Sven. J. Hammarling, The Numerical Algorithms Group, Ltd., Wilkinson House, Jordan Road, Oxford OX2 8DR, United Kingdom

106. Michael T. Heath, National Center for Supercomputing Applications, 4157 Beckman Institute, University of Illinois, 405 N. Mathews Avenue, Urbana IL 618012300

107. Bruce Hendrickson, Sandia National Laboratories, Division 1422, Albuquerque, NM 87185

108. Nicholas Higham, Dept. of Mathematics, University of Manchester, 90 Half Edge Lane, Eccles, Manchester, M13 9PL, England

109. Alan Hindmarsh, Lawrence Livermore National Laboratory, P.O. Box 808, L-316, Livermore, CA 94551

110. Michael J. Holst, California Institute of Technology, Applied Mathematics 217-50, Pasadena, CA 91125

111. Fred Howes, Office of Scientific Computing, ER-7, Applied Mathematical Sciences, Office of Energy Research, Department of Energy, Washington, DC 20585

112. James M. Hyman, Los Alamos National Laboratory, MS B284, Group T-7, Los Alamos, NM 87545

113. Ilse Ipsen, Dept. of Mathematics, North Carolina State University, Raleigh, NC 27695-8205

114. Erxiong Jiang, Department of Mathematics, Fudan University, Shanghai 200433, People's Republic of China

115. Gary Johnson, Office of Scientific Computing, ER-7, Applied Mathematical Sciences, Office of Energy Research, Department of Energy, Washington, DC 20585

116. Bo Kågström, Institute of Information Processing, University of Umeå, S-901, 87 Umeå, Sweden

117. Malvyn H. Kalos, Cornell Theory Center, Engineering and Theory Center Bldg., Cornell University, Ithaca, NY 14853-3901

118. Hans Kaper, Mathematics and Computer Science Division, Argonne National Laboratory, 9700 South Cass Avenue, Bldg. 221, Argonne, IL 60439

119. Kenneth Kennedy, Dept. of Computer Science, Rice University, P.O. Box 1892, Houston, TX 77001

120. David Keyes, NASA Langley Research Center, ICASE, M/S L23C, Hampton, CA 23681-0001

121. David Kincaid, Center for Numerical Analysis, RLM Hall 13.150, University of Texas at Austin, Austin, TX 78713-8510

122. Jacko Koster, 42 Ave G. Coriolis, 31057 Toulouse Cedex, France

123. Alan Laub, ECE Department, University of California at Santa Barbara, Santa Barbara, CA 93106-9560 
124. Richard Lehoucq, Dept. of Computational and Applied Mathematics, P.O. Box 1892, Rice University, Houston, TX 77251-1892

125. James E. Leiss, Rt. 2, Box 142C, Broadway, VA 22815

126. Steven J. Leon, Department of Mathematics, University of Massachusetts, Old Westport Road, North Dartmouth, MA 02747

127. John G. Lewis, Boeing Computer Services, P.O. Box 24346, M/S 7L-21, Seattle, WA $98124-0346$

128. Thomas A. Manteuffel, Program in Applied Mathematics, Campus Box 526, University of Colorado, Boulder, CO 80309-0526

129. Osni Marques, 42 Ave G. Coriolis, 31057 Toulouse Cedex, France

130. Pontus Matstoms, University of Linkoping, MAI, 58183 Linkoping, Sweden

131. James McGraw, Lawrence Livermore National Laboratory, P.O. Box 808, M/S L-306 Livermore, CA 94550

132. Gerard Meurant, Centre d'Etudes de Limeil, Department MA, 94195 Villeneuve St. Georges, France

133. Juan Meza, Sandia National Laboratories, Division 8211, P.O. Box 969, Livermore, CA 94551-0969

134. Cleve Moler, The Mathworks, 24 Prime Park Way, Natick, MA 01760

135. Neville Moray, Dept. of Mechanical and Industrial Engineering, University of Illinois, 1206 West Green Street, Urbana, IL 61801

136. David Nelson, Director of Scientific Computing, ER-7, Applied Mathematical Sciences, Office of Energy Research, U.S. Dept. of Energy, Washington, DC 20585

137. Martin Ojangruen, 42 Ave G. Coriolis, 31057 Toulouse Cedex, France

138. Dianne P. O'Leary, Dept. of Computer Science, University of Maryland, College Park, MD 20742

139. Beth Ong, University of California at San Diego, Department of Mathematics, 9500 Gilman Drive, La Jolla, CA 92093-0112

140. James M. Ortega, Dept. of Applied Mathematics, Thornton Hall, University of Virginia, Charlottesville, VA 22901

141. Michael Overton, Courant Institute, New York University, 251 Mercer Street, New York, NY 10012-1185

142. Chris Paige, McGill University, School of Computer Science, McConnell Engineering Building, 3480 University Street, Montreal, Quebec Canada H3A 2A7

143. Beresford N. Parlett, Dept. of Mathematics, University of California, Berkeley, CA 94720

144. Joao Patricio, 42 Ave G. Coriolis, 31057 Toulouse Cedex, France

145. Linda R. Petzold, Dept. of Computer Science, University of Minnesota, 4-192 EE/CS Bldg, 200 Union Street S.E., Minneapolis, MN 55455-0159

146. Robert J. Plemmons, Dept. of Mathematics and Computer Science, Box 7311, Wake Forest University, Winston-Salem, NC 27109 
147. Claude Pommerell, AT\&T Bell Laboratories, 2C-548A, Murray Hill, NJ 079740636

148. James C. T. Pool, Deputy Director, Caltech Concurrent Supercomputing Facility, California Institute of Technology, Pasadena, CA 91125

149. Alex Pothen, Old Dominion University, Computer Science Department, Norfolk, VA $23529-0162$

150. Chiara Puglisi, 42 Ave G. Coriolis, 31057 Toulouse Cedex, France

151. Bill Pulleyblank, IBM Research Division, P.O. Box 218, Yorktown Heights, NY 10598-0218

152. Padma Raghavan, Department of Computer Science, 657B Buehler Hall, University of Tennessee, Knoxville, TN 37996-1301

153. Lothar Reichel, Dept. of Mathematics, Kent State University, Kent, OH 44242

154. John Reid, Rutherford Appleton Laboratory, Oxon 0X11OQX, England

155. Werner C. Rheinboldt, Dept. of Mathematics and Statistics, University of Pittsburgh, Pittsburgh, PA 15260

156. Axel Ruhe, Department of Computer Science, Chalmers, S-41296 Göteborg, Sweden

157. Youcef Saad, Dept. of Computer Science, University of Minnesota, 4-192 EE/CS Bldg, 200 Union Street S.E., Minneapolis, MN 55455-0159

158. Faisal Saied, Department of Computer Science, 1304 W. Springfield Ave., University of Illinois at Urbana-Champaign, Urbana, IL 61801

159. Ahmed H. Sameh, Dept. of Computer Science, University of Minnesota, 4-192 EE/CS Bldg, 200 Union Street S.E., Minneapolis, MN 55455-0159

160. Michael Saunders, Systems Optimization Laboratory, Operations Research Dept., Stanford University, Stanford, CA 94305

161. Paul Saylor, Department of Computer Science, 1304 W. Springfield Ave., University of Illinois at Urbana-Champaign, Urbana, IL 61801

162. Hans Schneider, University of Wisconsin, Mathematics/Van Vleck Hall, 480 Lincoln Drive, Madison, WI 53706-1388

163. Robert Schreiber, RIACS, Mail Stop 230-5, NASA Ames Research Center, Moffet Field, CA 94035

164. Martin Schultz, Department of Computer Science, Yale University, P.O. Box 2158, Yale Station, New Haven, CT 06520

165. David S. Scott, Intel Scientific Computers, 15201 N.W. Greenbrier Parkway, Beaverton, OR 97006

166. Jeff Scroggs, Department of Mathematics, North Carolina State University, Box 8205, Raleigh, NC 27695

167. John Shadid, Sandia National Laboratories, Parallel Computer Science Division, Division 1421, Albuquerque, NM 87185

168. Bob Skeel, Department of Computer Science, 1304 W. Springfield Ave., University of Illinois at Urbana-Champaign, Urbana, IL 61801 
169. Danny Sorensen, Dept. of Mathematical Sciences, Rice University, P.O. Box 1892, Houston, TX 77251

170. Gerhard Starke, Universität Karlsruhe, Institut fur Praktische Mathematik, Englerstrasse 2, D-76128 Karlsruhe, Federal Republic of Germany

171. G.W. Stewart, Dept. of Computer Science, University of Maryland, College Park, MD 20742

172. Zdenek Strakos, Czech Academy of Sciences, Pod vod. vezi 2, 18207 Praha 8, Czech Republic

173. Gilbert Strang, Massachusetts Institute of Technology, Room 2-240, Cambridge, MA $02319-4557$

174. Ji-guang Sun, Institute for Information Processing, University of Umeå, S-901, 87 Umeå, Sweden

175. Xiobai Sun, Argonne National Laboratory, Bldg. 221, MCS Division, 9700 South Cass Ave., Argonne, IL 60439

176. Toshio Suzuki, Department of Mathematics, Yamanashi University, Kofu 400 , Japan

177. Paul N. Swartztrauber, National Center for Atmospheric Research, P.O. Box 3000, Boulder, CO 80307

178. Daniel Szyld, Dept. of Mathematics, Temple University, TU038-16, Philadelphia, PA $19122-2585$

179. Philippe Toint, Department of Mathematics, Rue de Bruxelles 61, B-5000 Namur, Belgium

180. Vincent Toumazou, 42 Ave G. Coriolis, 31057 Toulouse Cedex, France

181. Anne Trefethen, Dept. of Computer Science, Cornell University, Upson Hall, Ithaca, NY 14853

182. Nick Trefethen, Dept. of Computer Science, Cornell University, Upson Hall, Ithaca, NY 14853

183. Raymond S. Tuminaro, Sandia National Laboratories, Mail Stop 1110, P.O. Box 5800 , Albuquerque, NM 87185

184. Henk van der Vorst, Dept. of Mathematics, Universiteit Utrecht, P.O. Box 80010, 3508 TA, Utrecht, The Netherlands

185. Paul Van Dooren, Universite Catholique de Louvain, Centre for Systems Engineering and Applied Mechanics (CESAME), Batiment Euler (Rm A.119), 4, avenue Georges Lemaitre, B-1348 Louvain-la-Neuve, Belgium

186. Sabine Van Huffel, Katholieke Universiteit Leuven, ESAT Laboratory, Kardinaal Mercierlaan 943001 Heverlee, Belgium

187. Charles Van Loan, Dept. of Computer Science, Cornell University, Ithaca, NY 14853

188. Jim Varah, Centre for Integrated Computer Systems Research, University of British Columbia, Office 2053-2324 Main Mall, Vancouver, British Columbia V6T 1W5, Canada 
189. Richard S. Varga, Department of Mathematics, Kent State University, Kent, OH 44242

190. Andy Wathen, School of Mathematics, University Walk, Bristol University, Bristol BS8 1TW, United Kingdom

191. Mary F. Wheeler, Rice University, Dept. of Mathematical Sciences, P.O. Box 1892, Houston, TX 77251

192. Andrew B. White, Computing Division, Los Alamos National Laboratory, P.O. Box 1663, MS-265, Los Alamos, NM 87545

193. David Young, University of Texas, Center for Numerical Analysis, RLM 13.150, Austin, TX 78731-8510

194. Hongyuan Zha, Department of Computer Science and Engineering, 220 Pond Laboratory, The Pennsylvania State University, University Park, PA 16802-6106

195. Zahari Zlatev, Hagens Alle 28, DK-2900 Hellerup, Copenhagen, Denmark

196. Office of Assistant Manager for Energy Research and Development, U.S. Dept. of Energy, Oak Ridge Operations Office, P.O. Box 2001 Oak Ridge, TN 37831-8600

197-198. Office of Scientific \& Technical Information, P.O. Box 62, Oak Ridge, TN 37830 